\section{Skin Reaction Induced by Aldehydes for Food Flavoring Agents}

\author{
Kazuhito Watanabe, Midori Matsuda, \\ Shizuka Furuhashi, Toshiyuki Kimura, \\ Tamihide Matsunaga, and Ikuo Yamamoto* \\ Department of Hygienic Chemistry, Faculty of Pharmaceutical \\ Sciences, Hokuriku University, Kanazawa 920-1181, Japan \\ (Received February 1, 2001; Accepted February 22, 2001)
}

Guinea pigs were sensitized intracutaneously for 7 days ( $0.1 \mathrm{ml}$ of $1 \%$ aldehyde solution/day) with nine aldehydes ( $p$-anisaldehyde, benzaldehyde, citral, ethylvanillin, furfural, $\boldsymbol{n}$-octanal, $\boldsymbol{l}$-perillaldehyde, piperonal and vanillin) which are used as the food flavoring agents. Three weeks later, each aldehyde was injected by different concentrations (0.25-1.0\%). Skin reactions were observed $24 \mathrm{hr}$ after the injection of the aldehydes. All aldehydes tested exhibited a positive skin reaction, indicating that these aldehydes are capable of inducing an allergic reaction.

Key words — aldehyde, allergy, food additive, flavoring agent, guinea pig

\section{INTRODUCTION}

Aldehydes are known to be reactive, forming a Schiff base with an amino group in proteins. Thus, modification of protein molecules may cause the production of an antigen for an immuno-reaction. There are many reports on the sensitization of formaldehyde, which is used as a preservative in cosmetic products and industrial materials. ${ }^{1)}$ Sensitization to benzaldehyde, cinnamic aldehyde and phenylacetaldehyde has been repoprted in humans. ${ }^{2,3)}$ Aldehyde is formed as an intermediate metabolite of alkyl groups and primary alcohols. Shimeno and Yoshimura $^{4)}$ have reported that an aldehyde metabolite is a key intermediate in aminopyrine allergy. A line of evidence indicates that sensitization is a common property of the aldehydes.

In Japan, sixteen aldehydes are permitted for use as food flavoring agents. It is, therefore, important

\footnotetext{
*To whom correspondence should be addressed: Faculty of Pharmaceutical Sciences, Hokuriku University, Ho-3 Kanagawamachi, Kanazawa 920-1181, Japan. Tel.: +81-76-229-6220; Fax: +81-76-229-6221; E-mail: i-yamamoto@hokuriku-u.ac.jp
}

to assess whether these aldehydes induce allergic reaction including atopic dermatitis. The present study describes skin reactions in guinea pigs induced by aldehydes for food flavoring agents.

\section{MATERIALS AND METHODS}

Chemicals and Animals $-p$-Anisaldehyde, citral, ethylvanillin, $n$-octanal, piperonal and vanillin were obtained from Wako Pure Chem. Ind. (Osaka, Japan); benzaldehyde, furfural and $l$-perillaldehyde were purchased from Nacalai Tesque (Kyoto, Japan), Tokyo Kasei Kogyo Co. (Tokyo, Japan) and Aldrich Chem. Co., Inc. (U.S.A), respectively. Other chemicals used were obtained from Wako Pure Chem. Ind. (Osaka, Japan). Hartley male guinea pigs (8 weeks old, 300-400 g) were purchased from Sankyo Lab. (Toyama, Japan) and were given water and food $a d$ libitum.

Sensitization and Skin Reaction —— The animals were sensitized by the method described previously, with a slight modification. ${ }^{5,6)}$ Each group consisted of 3 to 4 animals. All aldehydes ( $p$-anisaldehyde, benzaldehyde, citral, ethylvanillin, furfural, $n$ octanal, $l$-perillaldehyde, piperonal and vanillin) were dissolved or suspended in saline containing $1 \%$ Tween 80. For sensitization, the animals were injected with $0.1 \mathrm{ml}$ of the aldehyde solution $(1.0 \%)$ intracutaneously on both sides of the abdomen for 7 days. Control animals received $0.1 \mathrm{ml}$ of saline- $1 \%$ Tween 80 solution intracutaneously instead of the aldehyde solution. Three weeks later, elicitation experiments were carried out with an injection of 0.1 $\mathrm{ml}$ of $0.25-1.0 \%$ aldehyde solution or saline containing $1 \%$ Tween 80 as the control. The abdomen of each animal was depilated with a hair remover (EBA cream, Tokyo-Tanabe Pharm. Co., Ltd., Tokyo, Japan) prior to the elicitation of a skin reaction. Skin reactions were graded $24 \mathrm{hr}$ after the injection of aldehydes or the vehicle as follows: 0 to $1.0 \mathrm{~mm}-, 1.0$ to $2.0 \mathrm{~mm} \pm, 2.0$ to $4.0 \mathrm{~mm}+, 4.0$ to $6.0 \mathrm{~mm}++, 6.0$ to $8.0 \mathrm{~mm} \mathrm{+++.5)}$

\section{RESULTS AND DISCUSSION}

Figure 1 shows a typical photo taken $24 \mathrm{hr}$ after elicitation with $p$-anisaldehyde. The photo clearly indicates that $p$-anisaldehyde induced a positive skin reaction. Figure 2 indicates the dependence of the size of the erythema on the concentration of the al- 


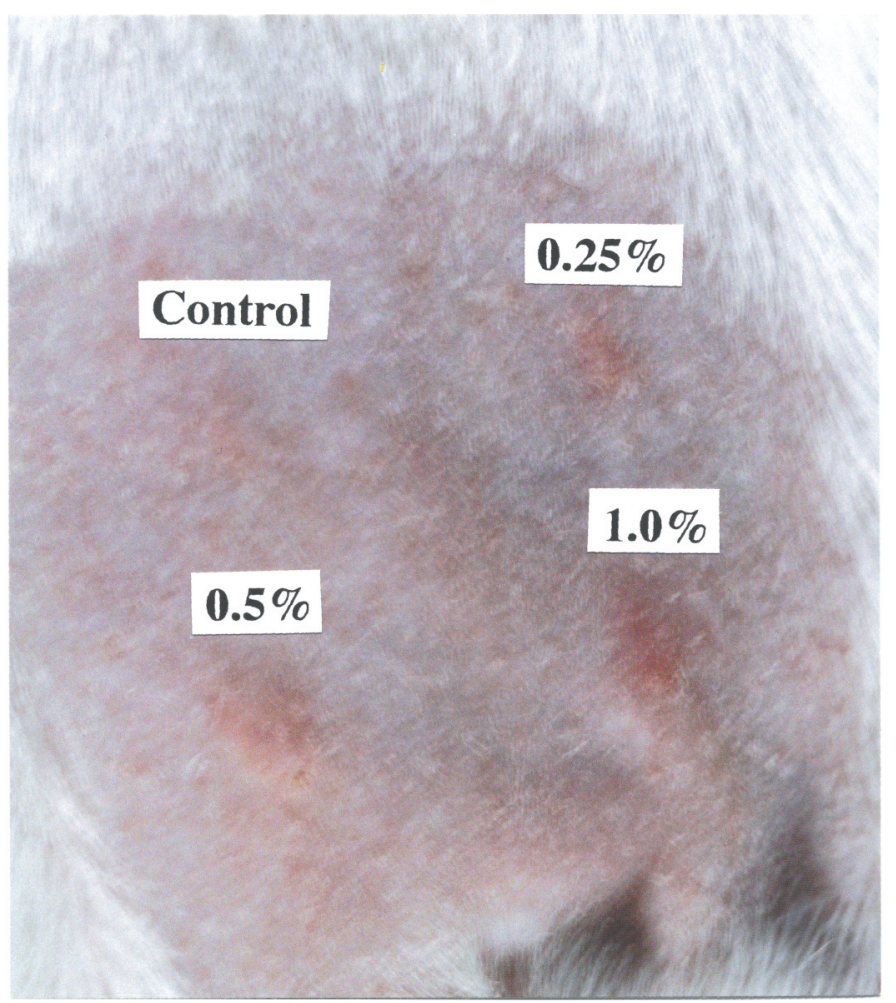

Fig. 1. Skin Reaction Induced by $p$-Anisaldehyde in Guinea Pig

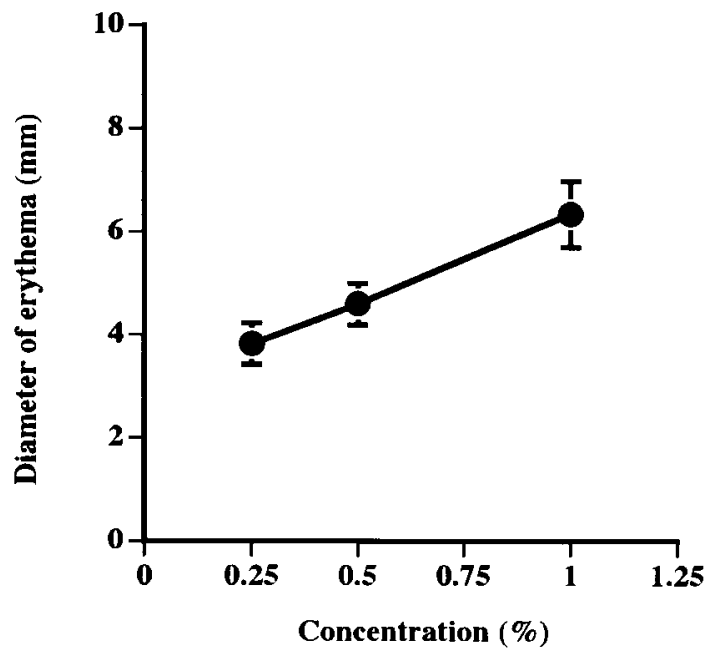

Fig. 2. Concentration-Dependence on Erythema Induced by $p$ Anisaldehyde

Each point represents the mean S.E. \pm from 4 animals.

dehyde injected. The skin reaction (diameter of erythema) progressively increased as the concentration of aldehyde increased. Table 1 summarizes the skin reactions induced by various aldehydes for food flavoring agents. A positive skin reaction was ob- served in all aldehydes tested in the present study, except that two of three animals treated with benzaldehyde and furfural, and one of three animals treated with citral showed no positive skin reaction. Among them, $p$-anisaldehyde, $n$-octanal and ethylvanillin exhibited relatively higher sensitizing properties. No skin reaction was observed after the injection of saline containing $1 \%$ Tween 80 .

Cinnamic aldehyde, a food flavoring agent, is known to be a contact allergen. ${ }^{7)}$ Weibel et al. ${ }^{8)}$ suggested that cinnamic aldehyde is an allergen, while cinnamic alcohol and cinnamic acid are transformed to active forms prior to the occurrence of contact allergic reactions. Ferguson and Beck ${ }^{9)}$ reported that vanilla containing vanillin in a lip salve produced contact sensitivity in human. These findings and the present results suggest that some aldehydes for food flavoring agents are allergens and have sensitizing properties. The risk of chronic ingestion of these aldehydes must be elucidated in relation to their metabolic studies.

Acknowledgments A part of this work was supported by the Special Research Fund of Hokuriku University. 
Table 1. Skin Reaction Induced by Aldehydes for Food Flavoring Agents

\begin{tabular}{|c|c|c|c|c|c|}
\hline \multirow[t]{2}{*}{ Aldehydes } & & \multicolumn{4}{|c|}{ Concentration used for skin reaction } \\
\hline & & 0 (saline-Tween80) & $0.25 \%$ & $0.5 \%$ & $1.0 \%$ \\
\hline \multirow[t]{4}{*}{$p$-Anisaldehyde } & 1 & \pm & + & ++ & +++ \\
\hline & 2 & - & + & ++ & ++ \\
\hline & 3 & - & ++ & ++ & ++ \\
\hline & 4 & - & + & + & +++ \\
\hline \multirow[t]{3}{*}{ Benzaldehyde } & 1 & - & - & - & - \\
\hline & 2 & - & - & - & - \\
\hline & 3 & - & ++ & +++ & +++ \\
\hline \multirow[t]{3}{*}{ Citral } & 1 & - & - & - & - \\
\hline & 2 & - & - & \pm & + \\
\hline & 3 & - & ++ & ++ & +++ \\
\hline \multirow[t]{3}{*}{ Ethylvanillin } & 1 & - & - & ++ & +++ \\
\hline & 2 & - & +++ & +++ & +++ \\
\hline & 3 & \pm & + & + & ++ \\
\hline \multirow[t]{3}{*}{ Furfural } & 1 & - & - & - & - \\
\hline & 2 & - & - & - & - \\
\hline & 3 & - & + & +++ & +++ \\
\hline \multirow[t]{3}{*}{$n$-Octanal } & 1 & - & + & ++ & +++ \\
\hline & 2 & - & ++ & ++ & +++ \\
\hline & 3 & \pm & +++ & +++ & +++ \\
\hline \multirow[t]{3}{*}{$l$-Perillaldehyde } & 1 & - & - & - & + \\
\hline & 2 & - & + & ++ & +++ \\
\hline & 3 & - & + & ++ & +++ \\
\hline \multirow[t]{3}{*}{ Piperonal } & 1 & - & - & - & ++ \\
\hline & 2 & - & + & ++ & ++ \\
\hline & 3 & \pm & \pm & ++ & +++ \\
\hline \multirow[t]{3}{*}{ Vanillin } & 1 & - & + & + & ++ \\
\hline & 2 & - & + & + & ++ \\
\hline & 3 & \pm & \pm & ++ & +++ \\
\hline
\end{tabular}

\section{REFERENCES}

1) Agner, T., Flyvholm, M. A. and Menne, T. (1999) Formaldehyde allergy: a follow-up study. Am. $J$. Contact Dermat., 10, 12-17.

2) Fregert, S. (1970) Sensitization to phenylacetaldehyde. Dermatologica, 141, 11-14.

3) Seite-Bellezza, D., Sayed, F. and Bazex, J. (1994) Contact urticaria from cinnamic aldehyde and benzaldehyde in a confectioner. Contact Dermatitis, $\mathbf{3 1}$, 272-273.

4) Shimeno, H. and Yoshimura, H. (1972) Possible implication of an aldehyde metabolite in aminopyrine allergy. Xenobiotica, 2, 461-468.

5) Yamamoto, I. and Eyanagi, R. (1977) Positive skin reaction induced by $4,4^{\prime}$-azoxybenzenedisulfonamide in relationship to the sulfanilamide allergy. Int. Arch.
Allergy Appl. Immun., 54, 538-541.

6) Eyanagi, R., Yoshida, K. and Yamamoto, I. (1979) Experimental studies on the antigenecity of some metabolites and related compounds of sulfanilamide. Yakugaku Zasshi, 99, 458-465.

7) Johansen, J. D., Andersen, K. E., Rastogi, S. C. and Menne, T. (1996) Threshold responses in cinnamic aldehyde-sensitive subjects: results and methodological aspects. Contact Dermatitis, 34, 165-171.

8) Weibel, H., Hansen, J. and Andersen, K. E. (1989) Cross-sensitization patterns in guinea pigs between cinnamyl aldehyde, cinnamyl alcohol and cinnamic acid. Acta Derm. Venereol., 69, 302-307.

9) Ferguson, J. E. and Beck, M. H. (1995) Contact sensitivity to vanilla in a lip salve. Contact Dermatitis, 33, 352 . 